\title{
Automated classification of primary progressive aphasia subtypes from narrative speech samples
}

\author{
Fraser K. C., Meltzer J. A., Graham N. L., Leonard C., Hirst G., \\ Black S. E. \& Rochon E.
}

\author{
Version Post-print/accepted manuscript \\ Citation Fraser, K.C., Meltzer, J.A., Graham, N.L., Leonard, C., Hirst, G., Black, \\ (published version) S.E., Rochon, E. Automated classification of primary progressive \\ aphasia subtypes from narrative speech transcripts. Cortex. 2014; 55:43- \\ 60. DOI: $10.1016 /$ j.cortex.2012.12.006. \\ Copyright/License \\ (c) (1) $\odot$ This work is licensed under the Creative Commons \\ BY NC ND Attribution-NonCommercial-NoDerivatives 4.0 \\ International License. To view a copy of this license, visit \\ http://creativecommons.org/licenses/by-nc-nd/4.0/.
}

How to cite TSpace items

\begin{abstract}
Always cite the published version, so the author(s) will receive recognition through services that track citation counts, e.g. Scopus. If you need to cite the page number of the author manuscript from TSpace because you cannot access the published version, then cite the TSpace version in addition to the published version using the permanent URI (handle) found on the record page.
\end{abstract}

This article was made openly accessible by $U$ of $T$ Faculty. Please tell us how this access benefits you. Your story matters. 


\section{Automated classification of primary progressive aphasia subtypes from narrative speech samples}

Kathleen C. Fraser ${ }^{1}$, Jed A. Meltzer ${ }^{2}$, Naida L. Graham ${ }^{3,4}$, Carol Leonard ${ }^{5}$, Graeme Hirst ${ }^{1}$, Sandra E. Black ${ }^{6,7}$, and Elizabeth Rochon ${ }^{3,4}$

${ }^{1}$ Department of Computer Science, University of Toronto, Toronto, Ontario, Canada

${ }^{2}$ Rotman Research Institute, Baycrest Centre, Toronto, Ontario, Canada

${ }^{3}$ Department of Speech-Language Pathology, University of Toronto, Toronto, Ontario, Canada

${ }^{4}$ Toronto Rehabilitation Institute, Toronto, Ontario Canada

${ }^{5}$ School of Rehabilitation Sciences, University of Ottawa, Ottawa, Ontario, Canada

${ }^{6}$ L.C. Campbell Cognitive Neurology Research Unit, Sunnybrook Health Sciences Centre, Toronto, Ontario, Canada

${ }^{7}$ Department of Medicine (Neurology), University of Toronto, Toronto, Ontario, Canada

Corresponding author: Kathleen C. Fraser (kfraser@cs.toronto.edu)

Department of Computer Science

10 King's College Road, Room 3302

Toronto, Ontario, Canada

M5S 3A6.

Telephone: 1-647-232-6546. Fax: 1-416-978-1455. 


\begin{abstract}
In the early stages of neurodegenerative disorders, individuals may exhibit a decline in language abilities that is difficult to quantify with standardized tests. Careful analysis of connected speech can provide valuable information about a patient's language capacities. However, this type of analysis has been limited in the past by its time-consuming nature. In this study, we present a method for evaluating and classifying connected speech in primary progressive aphasia (PPA) using computational techniques. Syntactic and semantic features are automatically extracted from transcriptions of narrative speech for three groups: semantic dementia (SD), progressive nonfluent aphasia (PNFA), and healthy controls. Features that vary significantly between the groups are used to train machine learning classifiers, which are then tested on held-out data. We achieve accuracies well above baseline on the three binary classification tasks. An analysis of the influential features shows that the computationally extracted measures agree well with previous findings in the PPA literature, suggesting that this method could have valuable applications to diagnosis and research.
\end{abstract}

Keywords: semantic dementia; progressive nonfluent aphasia; narrative speech; natural language processing; machine learning 


\section{Introduction}

Primary progressive aphasia (PPA) is a dementia syndrome, resulting from neurodegenerative disease, in which language impairment is the earliest and most salient feature. It is widely accepted that there are three variants of PPA (Gorno-Tempini et al., 2004): progressive nonfluent aphasia (PNFA), progressive fluent aphasia, often referred to as semantic dementia (SD) due to the pervasive semantic impairment, and logopenic progressive aphasia. PNFA is characterized by nonfluent, hesitant, effortful speech, with word-finding difficulty; in addition, agrammatism and/or apraxia of speech are considered to be core features. In $\mathrm{SD}$, there is severe anomia, although spoken output remains fluent, well-articulated, and grammatically correct, with normal prosody. The logopenic variant is associated with hesitant speech, obvious word-finding difficulty, and intact word repetition but poor repetition of phrases and sentences; this variant is not a focus of the present study and therefore will not receive further attention. Until recently, most systematic investigations of spoken output in PPA focused on single word production (naming, reading, repetition), but there is now a small literature that examines production of connected speech. Difficulty with conversing is often a presenting complaint in PPA, and diagnostic criteria describe the nature of the impairment in spoken output that is indicative of each variant (Gorno-Tempini et al., 2004). Because impairment in connected speech is the essence of PPA, thorough characterization seems essential. The main hurdle to date has been the laborious process required for transcription and systematic analysis of connected speech. Nevertheless, progress has been made and we are beginning to understand the characteristics of language production in connected speech in each variant of PPA.

Patients with PNFA tend to have reduced output in comparison with control participants: it has been shown that they produce fewer words (Graham et al., 2004; Meteyard and Patterson, 2009), fewer clauses (Meteyard and Patterson, 2009), shorter phrase length (Knibb et al., 2009), and a shorter mean length of utterance (Ash et al., 2006; Thompson et al., 2012). As well, their speech rate is slower and their speech is less informative than that of 
controls (Ash et al., 2006; Graham et al., 2004; Knibb et al., 2009; Thompson et al., 2012; Wilson et al., 2010). The results with respect to grammatical competency have been less consistent. Some studies have suggested that patients with PNFA may use simplified syntax, but that outright grammatical errors are rare (Graham et al., 2004; Meteyard and Patterson, 2009; Patterson and MacDonald, 2006). This work also documented normal ratios of nouns to verbs and content words to function words, suggesting that grammatical production is preserved to a large extent. In contrast, other work documented increased grammatical errors in PNFA (Knibb et al., 2009), reduced production of grammatically correct sentences (Thompson et al., 2012), and impairment in production of verb inflection and argument structure (Thompson et al., 2012). Part of this discrepancy in results may have arisen from differences in participant selection, as Knibb et al.'s group may have included logopenic patients, and Thompson et al.'s nonfluent group all had clinically identified agrammatism.

The work on production of connected speech in patients with SD has demonstrated that they tend to use words which are higher in frequency but less specific than the words used by controls (Meteyard and Patterson, 2009). They also produce more pronouns, as well as more pronouns with ambiguous referents (Kavé et al., 2007; Meteyard and Patterson, 2009; Patterson and MacDonald, 2006; Wilson et al., 2010). Thus, it is not surprising that the speech of SD patients has been shown to be less informative than that of controls (Ash et al., 2006; Kavé et al., 2007; Meteyard and Patterson, 2009). There is also a tendency to use nouns and verbs which are higher in frequency than those used by controls (Bird et al., 2000). The rate of syntactic and phonological errors is no higher than controls (Sajjadi et al., 2012; Wilson et al., 2010), but the level of syntactic ability is a matter for debate. Some studies have documented normal ratios of content words to function words and ratios of nouns to verbs (Meteyard and Patterson, 2009; Sajjadi et al., 2012), suggesting normal grammatical production, but others found that both of these ratios were abnormal (Bird et al., 2000; Garrard and Forsyth, 2010; Thompson et al., 2012). Similarly, there has been inconsistency with respect to the findings regarding speech rate, which has been found to be 
both normal (Bird et al., 2000; Garrard and Forsyth, 2010; Meteyard and Patterson, 2009; Thompson et al., 2012) and reduced (Ash et al., 2006; Sajjadi et al., 2012; Wilson et al., 2010). Interestingly, Sajjadi et al. (2012) found that SD patients do not exhibit frequent circumlocution, despite numerous clinical descriptions to the contrary.

In this study, we examine narrative speech in PNFA and SD. In contrast to the studies reviewed above, to gain maximum information we used methods from natural language processing, which involves the use of software to analyze speech samples, or in our case, transcriptions of speech samples. These methods enable, for example, part-of-speech (POS) tags to be automatically assigned to words in a text using a statistical POS tagger. Others have begun to use these methods to analyze spoken output in dementia. For example, Roark et al. (2011) compared automatic and manual methods for determining syntactic structure of spoken output, and demonstrated that the automatic method was sufficiently accurate to enable identification of syntactic complexity measures that distinguished between healthy participants and those with mild cognitive impairment.

Peintner et al. (2008) have adopted this approach. They studied speech from patients with frontotemporal dementia (FTD), and used a subset of extracted features as input to machine learning classifiers to classify each participant as belonging to the PNFA, SD, or behavioural variant FTD groups, or as a control. A similar procedure was followed by Jarrold et al. (2010) when they used machine learning algorithms to classify transcriptions of speech from participants with pre-symptomatic Alzheimer's disease, mild cognitive impairment, or depression. Both studies had some success with classification based on samples of connected speech, but they are limited in that they do not report which features were able to reliably distinguish between patient groups.

The present study had two aims. The first was to develop a machine learning classifier that would analyze speech samples and be able to distinguish between control participants and participants with PNFA or SD, as well as between the two patient groups. The other aim of this study was to identify the automatically extracted features that best distinguish 
the groups, and to compare this with results in the literature that are based on traditional (manual) analysis methods. Identification of the distinguishing features is important for improved detection and differentiation of the variants of PPA. Furthermore, beyond diagnosis, improved quantitative characterization of PPA symptoms provides a basis for tracking disease severity, which can inform attempts to develop therapeutic interventions in PPA and in other disorders that affect speech.

\section{Participants and Methods}

\subsection{Participants}

Our participants comprised 24 patients diagnosed with either the fluent (SD) or nonfluent variant (PNFA) of primary progressive aphasia (PPA), and 16 age- and education-matched healthy controls. The patient group is an unselected sample of patients with SD or PNFA, except that participants who were unable to complete the narrative task $(n=7)$ were excluded: 2 PNFA patients had incomprehensible speech, 1 PNFA patient said nothing, 1 SD patient refused to attempt the task, and the responses of 1 PNFA and 2 SD patients did not include any of the story that they were asked to tell, but instead comprised statements of how they could not remember the story. Participants with PPA were recruited through three memory clinics in Toronto and each was diagnosed by an experienced behavioural neurologist. There were a further 7 patients in the cohort who were diagnosed with logopenic PPA, but this group was not included in the present study due to its small size. Control participants were recruited from a volunteer participant pool. All participants were native speakers of English, or completed some of their education in English. Exclusion criteria included a known history of drug or alcohol abuse, or a history of neurological or major psychiatric illness.

The study was approved by the Research Ethics Boards of all the hospitals involved in

recruitment, as well as the board at the University of Toronto. Written informed consent 
was obtained from all participants.

Diagnosis was based on history, neuroimaging, neurological examination and neuropsychological testing, and all patients met current criteria for PPA (Gorno-Tempini et al., 2011). Patients with the fluent variant exhibited grammatically correct fluent speech, with word finding difficulties. Those with the nonfluent variant had effortful, halting speech with anomia, although not all exhibited clear agrammatism in production or clear apraxia of speech on formal testing. Demographic data are listed in Table 1.

All participants underwent a battery of neuropsychological and linguistic tests as part of a longitudinal study of PPA which is being conducted in the Department of Speech-Language Pathology of the University of Toronto. The neuropsychological test information is reported in Table 1. The level of general cognitive functioning was measured using the Mini-Mental State Examination (Folstein et al., 1975) and the Dementia Rating Scale-R (Jurica et al., 2001). The two patient groups did not differ on these tests, but were both impaired relative to controls. In keeping with the diagnosis of PPA, both patient groups performed poorly on a test of picture naming (Boston Naming Test, Kaplan et al. (2001)) and on category fluency for animals (where participants are asked to name all the animals they can think of in 1 minute). Impairment in syntactic comprehension is a known feature of PNFA and indeed was exhibited by the PNFA group studied here; this ability was measured using the Test for the Reception of Grammar (Bishop, 2003), and the nonfluent group (only) performed significantly worse than controls. Impairment in single word comprehension is an established feature of SD; both of our patient groups were impaired on single word comprehension, but as expected, the SD patients performed significantly worse than the PNFA patients (Peabody Picture Vocabulary Test, Dunn and Dunn (1997)). Consistent with the diagnosis of PPA, performance was generally better on nonverbal tests. The PNFA group was mildly impaired on copying the Rey Complex Figure (Rey, 1941) while the SD group showed normal performance. On another measure of visuospatial functioning, the cube analysis subtest from the Visual Object and Space Perception Battery (Warrington and James, 1991), both 
patient groups performed normally. Similarly, performance on nonverbal episodic memory was normal for both patient groups; this was assessed by asking participants to recall the Rey Complex Figure 30 minutes after copying it. Finally, nonverbal reasoning was relatively preserved, although it was mildly impaired for the PNFA group (only) (Raven's Coloured Progressive Matrices, Raven (1962)).

\subsection{Narrative task}

Speech samples were elicited by having participants tell the Cinderella story, as in Saffran et al. (1989). To prompt their memories for the story, participants were given as much time as they needed to examine a picture book illustrating the story. When each participant had finished looking at the pictures, and the book had been removed, the examiner said "Now you tell me the story. Include as much detail as you can and try to use complete sentences." After letting the participant speak for as long as he or she wished, if the story was incomplete, general encouragement for more speech was given, for example, "Good, tell me more about that", "What happens next", "Go on", etc. At no time were specific questions or prompts given. The narratives were recorded on a digital audio recorder for subsequent verbatim transcription. Transcription was done in accordance with the procedures used in the Quantitative Production Analysis (Berndt et al., 2000), with the exception that punctuation and sentence initial capitalization were used. Brief pauses were marked with commas, while pauses longer than 1 second were timed and the length of the pause was noted (e.g., (2 sec)). Sentence boundaries were marked with full stops. Placement of sentence boundaries and commas was guided by semantic, syntactic and prosodic features, using a method essentially identical to that described by Thompson et al. (2012). When utterance boundaries were ambiguous, we created shorter utterances (as did Thompson et al. (2012) and Wilson et al. (2010)). Fillers such as "um" and "uh" were transcribed (and analyzed), but were not included in the total word count. Repetitions, false starts and stutters were transcribed, but only repetitions and false starts were included in the total word count. Neologisms were 
transcribed with IPA, and words/passages which were incomprehensible were marked with \#\#\#. Neologisms and incomprehensible speech were not included in the automated analyses or in the word counts as we could not be certain how many words were represented; note, however, that incomprehensible passages were always brief. There were only rare instances of neologisms or incomprehensible speech: 3 participants (one in each group) had 2 occurrences each, while a further 3 participants had 1 occurrence (2 PNFA, 1 SD). The mean number of words (as well as standard deviation and range) in the speech samples for each group was: SD 402.2 (281.7, 78-934); PNFA 327.8 (145.5, 147-583); controls 427.7 (121.1, 265-620).

\subsection{Analysis of narrative speech}

The automatically extracted features are defined in Table 2 . The first feature is the number of words in the transcript. The subsequent 22 structural features (2-23) were calculated using Lu's L2 Syntactic Complexity Analyzer (Lu, 2010). Here we have modified features 3-9 to be normalized by the total number of words, to facilitate comparison between narratives of different lengths. Lu used these features to analyze the syntactic complexity of college-level English essays from Chinese students, but the software has also been used to analyze spoken language (Chen and Zechner, 2011).

The next four features (24-27) are also measures of syntactic complexity. Tree-based measures have been used to detect age-related cognitive decline (Cheung and Kemper, 1992) and mild cognitive impairment (Roark et al., 2011). Parse trees were constructed using the Stanford parser (Klein and Manning, 2003). The Yngve depth quantifies to what extent the syntactic structure of a sentence contains left-branching rather than right-branching phrases, which provides a comparison metric of syntactic complexity (Yngve, 1960). For detailed illustrations of how Yngve depth is quantified, see Sampson (1997); Cheung and Kemper (1992); Yngve (1960). We quantified Yngve depth as both mean depth over all words, the maximum depth in the sentence, and the total depth. For example, a sentence with an object-embedded relative clause such as The juice that the child spilled stained the 
rug is more left-branching than one with a subject-embedded relative clause such as The child spilled the juice that stained the rug (Stromswold et al., 1996). Using our procedures, the first sentence is assigned these values: [max depth: 3, mean depth: 1.67, total depth: 15], while the second sentence is assigned: [max depth: 2, mean depth: 1.11, total depth: $10]$.

Features 28-40 rely explicitly on the part-of-speech (POS) tags for each word in the sample, determined by using the Stanford POS tagger (Toutanova et al., 2003). Differences in the noun and verb production of SD and PNFA patients have been noted before (Harciarek and Kertesz, 2011). It has also been observed that PNFA patients are more likely to omit inflections and function words (Harciarek and Kertesz, 2011). Here, function words included determiners, pronouns, prepositions, conjunctions, particles, and modals.

Verbs can be categorized as being heavy or light, according to their semantic complexity. For example, light verbs like have or do can be used in such a wide variety of different contexts that they are similar in some ways to closed-class function words (Breedin et al., 1998). We used the same list of light verbs as Breedin et al. (1998), namely: be, have, come, go, give, take, make, do, get, move, and put. All verbs which are not on this list are considered to be heavy.

Features 41-52 measure frequency, imageability, age of acquisition, and familiarity. Frequency was calculated according to the SUBTL norms (Brysbaert and New, 2009), and the remaining three according to the combined Bristol norms and Gilhooly-Logie norms (Stadthagen-Gonzalez and Davis, 2006; Gilhooly and Logie, 1980). In addition to calculating the total averages, the measures were calculated for nouns and verbs independently, to explore any possible dissociations. The coverage for the frequency norms is excellent between .92 and .95 across the three groups. The coverage for the imageability, age of acquisition, and familiarity norms is significantly worse, ranging from .25 to .31 for all content words across the three groups. One reason for this is that the Bristol norms were specifically designed to exclude high frequency words, as the authors wanted to use words in "the fre- 
quency range most often sampled by psycholinguistic experiments" (Stadthagen-Gonzalez and Davis, 2006). This means that most of the words have frequencies between 1 and 100 counts per million. For example, nouns like thing (which has a frequency of 1088 counts per million in the SUBTL norms) and name (641 counts per million) are excluded, as well as verbs like go (3793 counts per million) and do (6135 counts per million). The coverage was measured per word type, as opposed to per token.

The last six features (53-58) are measures of fluency and vocabulary richness. One way to measure vocabulary size is by calculating the type-token ratio (TTR), which is the ratio of the number of word types to the total number of words in the sample. A TTR of 1.0 would mean that every word in the sample was unique; a low TTR would indicate that a lot of words were repeated. Filled pauses are measured by counting occurrences of the words um, uh, ah, and er, called "fillers" in Table 2. The words um and uh were also counted individually, because of research which suggests that they may be used to indicate major and minor pauses, respectively (Clark and Fox Tree, 2002). Finally, the speech rate has been shown to distinguish between PPA patients (regardless of subtype) and controls, with PPA patients showing a reduction in speech rate (Wilson et al., 2010). Here we consider an estimate of the speech rate, calculated by dividing the total speech sample time by the number of words produced by the patient.

Impaired speech can present difficulties for automatic language processing techniques, which have typically been developed for well-formed, written text. However, these methods represent a starting point for future development of more sophisticated techniques. To increase the probability of the structural analyses producing accurate results, our system first counts the filled pauses and then removes them from the transcript, as well removing short nonword tokens (e.g. stutters).

To test the results of our automatic methods against traditional manual methods, we had a human annotator perform part-of-speech tagging and calculate a subset of the parse measures for three randomly-chosen narratives from each patient group (22.5\% of the total 
data set). For part-of-speech tagging, we measured the agreement between the human annotator and the automatic tagger. The average agreement was $87.3 \%$ for the PNFA group, $89.2 \%$ for the SD group, and $91.9 \%$ for the control group.

In comparing the parse features, we were limited by the fact that Lu's program simply outputs counts for each measure, rather than the actual constituents being measured. This makes it impractical to use traditional parse measures such as the PARSEVAL measures (Manning and Schütze, 1999). Instead we had the annotator produce counts of different structures, just as the software does, and then compared the correlation between the two sets of counts. We examined only features which were found to be significant; namely clauses, dependent clauses, T-units, complex T-units, and coordinate phrases. The correlation coefficients for these measures are shown in Figure 3.

Despite the high correlations, we did find some discrepancies which should be noted. In general, the automatic system has high agreement with the human annotator when determining the number of clauses. However, it has difficulty labelling clauses as being either independent or dependent, especially when the clauses are not connected by a conjunction. In many such cases, the system counted more dependent clauses than the human annotator, which in turn affects the number of T-units and complex T-units. Given this apparently systematic error, the results from Lu's syntactic complexity analyzer must be interpreted with caution.

\subsection{Classification}

We trained machine learning classifiers to predict participants' diagnoses based on a set of features extracted automatically from speech transcripts. Including too many features risks overfitting the classifier to idiosyncrasies in the training set, resulting in poor generalization to new data points. Therefore, some process of feature selection is necessary. To select features on which to train the classifiers, we conducted a two-sample $t$-test on each feature between the two groups that were to be distinguished. All features that were significant at 
$p<0.05$ were used for classification. The values of the selected features make up a feature vector, which defines a point in feature space. The goal of a machine learning classifier is to take a feature vector as input, and output a class label (in this case, either SD, PNFA, or control). Three machine learning classifiers from the WEKA machine learning toolkit were compared (Hall et al., 2009).

Naïve Bayes is a classifier based on Bayes's theorem. It is called "naïve" because it makes the strong simplifying assumption that all of the features are conditionally independent given the class. The classifier learns estimates for the class-conditional probabilities and priors for each class from the training data. In the classification stage, it uses Bayes's theorem to assign a data point to the class that maximizes the posterior probability. Naïve Bayes is widely used, even in cases where the independence assumption is known to be false, and often performs quite well. The rationale for this is that even though the probability estimates may be inaccurate, the classification results (which only depend on which probability is the highest, and not on the actual numbers) can be quite good (Manning et al., 2008).

In contrast to naïve Bayes, which attempts to model the classes themselves, logistic regression is a discriminative classifier which attempts to model the boundary between the classes instead. Logistic regression estimates the posterior probability directly from the training data. Research suggests that naïve Bayes may perform better in cases where there is not a large amount of training data ( $\mathrm{Ng}$ and Jordan, 2002). However, the benefit of logistic regression is that it does not assume the features are conditionally independent. Peintner et al. (2008) used logistic regression, along with two other classifiers not considered here, on various classification tasks involving FTD subtypes and healthy controls. They had mixed results, with logistic regression achieving the best results in two out of six cases.

Support vector machines (SVMs) are another type of linear discriminative classifier which have become very popular in natural language processing applications in the past several years (Manning et al., 2008). SVMs are a maximum margin classifiers, which means they find the decision boundary between two classes that maximizes the margin between the two 
classes. In other words, they maximize the distance between the decision boundary and the nearest data points. If the data are not linearly separable, then the algorithm tries to maximize the margin while also minimizing the misclassification error (Manning et al., 2008).

The classifiers were evaluated based on classification accuracy, or the total proportion of narratives which were correctly classified. The evaluation was performed using leave-one-out cross validation. In this procedure, one data point is left out, and the classifier is trained on the remaining data. The left-out data point can then be used as an unbiased test set. This procedure is repeated until each data point has been left out once, and the performance is averaged.

\section{Results}

We consider three separate classification tasks: (1) distinguishing between SD and controls; (2) distinguishing between PNFA and controls; and (3) distinguishing between SD and PNFA. The means and standard deviations for each attribute are compared in Tables 4 to 6. Group differences were measured using Welch's two-tailed, unpaired $t$-test, which does not assume that the two samples share the same variance. A significance level of $p<.05$ is indicated by a single asterisk, and $p<.01$ is indicated by a double asterisk. Because we are using the $t$-tests primarily as a means of feature selection, we do not adjust their significance levels for multiple comparisons. For each classification task, the set of significant features for that particular comparison formed the input vectors to the classifiers. The features were rescaled to have zero mean and unit variance before classification, to prevent features with large magnitudes (e.g. imageability) from dominating features with smaller magnitudes (e.g. fillers).

The features that were considered significant between the SD and control transcripts, and therefore used in that classification task, were: number of clauses, mean length of sentence, mean length of clause, T-units per sentence, total Yngve depth, mean Yngve depth, 
nouns, noun-verb ratio, noun ratio, demonstratives, adverbs, pronoun ratio, frequency, noun frequency, verb frequency, verb imageability, familiarity, noun familiarity, mean word length, and speech rate. (see Table 4). For the task of classifying PNFA versus controls, the significant features were: number of words, T-units per sentence, demonstratives, frequency, verb frequency, age of acquisition, noun age of acquisition, mean word length, and speech rate (see Table 5). In the case of SD versus PNFA, only five features were significant: dependent clauses per clause, noun frequency, familiarity, noun familiarity, and occurrence of "um" (see Table 6).

The classification accuracies are given in Table 7 . The baseline accuracies represent the accuracies that would be achieved by simply assigning every transcript to the larger of the two classes. That is, the baseline accuracy for SD $(n=10)$ versus controls $(n=16)$ would be achieved by simply classifying all the transcripts as controls $(16 / 26=.615)$. The two experimental scenarios involving patient groups versus control groups result in very high classification accuracies. The accuracies for classifying SD versus PNFA transcripts are not as high; however, they are well above the baseline for all three of the classifiers.

For comparison, we also evaluated the performance of the classifiers trained on all features as input, rather than just the features pre-selected by the $t$-tests. This method was expected to perform rather poorly due to overfitting. Although using more features may improve classification on the training set, it results in poor generalization to new data, as assessed with the cross-validation procedure. Indeed, performance in this case was lower than the results shown in Table 7: for SD versus controls, the accuracies ranged from .846 to .923, for PNFA versus controls the accuracies ranged from .700 to .800, and for SD versus PNFA they ranged from .625 to .667 . This illustrates the necessity of feature selection prior to classifier training.

Because the classification takes place in high-dimensional feature space, it is difficult to visualize the models produced by the classifiers. Instead, it is useful to visualize the classes in two dimensions by using some form of dimensionality reduction. Here we use the 
method of partial least squares, or PLS (Haenlein and Kaplan, 2004). PLS is similar to the well-known method of principal components analysis (PCA), except that PCA discovers the latent variables that best explain the variance in the attributes, while PLS discovers the latent variables that are most predictive of the response (in this case, the patient groups or class labels). In addition, PLS is appropriate when the number of attributes is high compared to the number of data points, which is the case here.

Scatter plots of the first two PLS components are shown in Fig. 1. The plot for SD versus controls shows relatively good separation between the groups, while the plot for PNFA versus controls has a small overlap between groups. The plot for SD versus PNFA shows more overlap, which is consistent with the reduced classification accuracies in that case.

Rather than analyze the factor loadings, which are not easily interpretable in PLS, we calculate selectivity ratios, which are closely related to the correlation between each attribute and the response (Kvalheim, 2010). A high selectivity ratio indicates that a feature is very influential with respect to the response. The details of calculating this measure are given by Kvalheim (2010); we used a pre-existing Matlab package to perform the analysis (Li, 2011). Figure 2 shows the selectivity ratios for each feature in the three cases. For ease of interpretation, each feature with a selectivity ratio of greater than a cut-off of 0.5 is given for each of the three classification problems. In the majority of cases, these are the same features which were found to be significant and included in the classifiers above. We expect there to be some discrepancies, as the individual $t$-tests do not take into account correlations between variables, while the PLS analysis does. Selectivity ratios for influential features may be reduced in the presence of a second feature highly correlated with the first, as the two share variance that predicts group membership. 


\section{Discussion}

In this study we set out to determine whether computational methods could reliably distinguish between healthy controls and patients with PPA in addition to differentiating the two patient groups, based upon samples of narrative speech. We found that even with relatively short samples of narrative speech (i.e., for machine learning purposes), classifiers were able to achieve this goal with a high degree of accuracy. In addition, we wished to determine how the automatically extracted features compared to previous findings in the literature with regards to these two subtypes. In general, we found that our procedure identified many of the same features that have been previously noted to differ between groups (e.g., word frequency, speech rate, demonstrative pronouns), with some surprising findings (e.g., the lack of syntactic features as differentiating ones) and some new features identified (e.g., adverbs and word length). We discuss these issues at greater length below.

\subsection{Classification}

Our results show that machine learning classifiers can distinguish between controls and each

of the two patient groups, SD and PNFA, with a high degree of accuracy. Although less accurate than in comparison to controls, they also distinguish well between the two patient groups. The performance of the classifiers varied across the three tasks: SVM achieved the highest accuracy for SD versus controls and PNFA versus controls, while naïve Bayes performed best for SD versus PNFA. Logistic regression achieved the second-highest accuracy in the first two cases, but was the worst at distinguishing between SD and PNFA. We also note the relatively high accuracy of naïve Bayes despite obvious correlations between the features in some cases. 


\subsection{Comparison with previous findings on PPA}

The PLS analysis identified the features that best predicted group membership. The selectivity ratios, together with the group means on each feature, provided valuable information on the characteristics of narrative speech in each group.

The features that best distinguished the SD patients from controls were higher frequency of words and of nouns in particular, higher familiarity, increased production of adverbs and demonstratives, and reduced word length and speech rate. The frequency of the nouns produced, which was higher in SD, was the feature with the highest selectivity ratio. The finding that overall word frequency also had a high selectivity ratio demonstrates the robust effect of frequency on language production in SD. Because noun frequency and overall frequency are correlated, each would have had an even higher selectivity ratio if the other had not been included in the model. It has been established that frequency has a pervasive influence on naming in SD (Lambon Ralph et al., 1998; Woollams et al., 2008), but this influence is less well documented in connected speech. Two studies which used picture description tasks observed that SD patients produced higher frequency nouns than controls (Bird et al., 2000; Wilson et al., 2010). Meteyard and Patterson (2009) did not evaluate frequency per se, but their analysis of structured interviews showed that patients with SD tended to replace content words with higher frequency (and less specific) words. We have found that patients with SD use higher frequency words overall, but that nouns are particularly affected.

The finding that the SD patients produced words with higher familiarity ratings than controls is consistent with findings from studies of naming (Lambon Ralph et al., 1998; Woollams et al., 2008), but to the best of our knowledge has not been previously documented in connected speech. It also fits with previous research demonstrating that SD patients' semantic knowledge is best preserved for familiar items (Funnel, 1996; Simons et al., 2001). Note that familiarity and frequency are correlated (Tanaka-Ishii and Terada, 2011), so indi-

vidually they would have had even higher selectivity ratios if only one of the features had been included in the model. 
The SD patients produced more adverbs and demonstratives than controls, and this distinguished the groups. The increased use of adverbs may at least partially reflect that fact that some participants in this patient group started many of the sentences in their narratives with "then" or "so", sometimes preceded by "and". Because "then" and "so" are classified as adverbs (describing when or why something happened), this inflates the count on these words. Repeated use of the same syntactic structure is compatible with the idea that SD patients are unable to produce the full range of syntactic structures (Benedet et al., 2006), but needs to be investigated further before firm conclusions can be drawn. The increased reliance on demonstrative pronouns, which comprise the words these, those, this, that, here, and there, may reflect the tendency of SD patients to make substitutions of less specific words (Meteyard and Patterson, 2009) and use vague terms (Kavé et al., 2007; Patterson and MacDonald, 2006); the automated analysis techniques used here did not enable us to evaluate whether these terms had clear referents when used. Previous studies have documented over-reliance on pronouns in SD (Kavé et al., 2007; Meteyard and Patterson, 2009; Patterson and MacDonald, 2006; Wilson et al., 2010), but to the best of our knowledge this is the first examination specifically of demonstrative pronouns.

The mean word length for the SD patients was slightly shorter than for controls, and this feature distinguished well between the groups. Word length has been shown to affect performance on naming in $\mathrm{SD}$, but has not been examined in connected speech. We attribute this small but significant effect of length to choice of words, rather than to difficulty with pronouncing long words. Many of the long words used by controls were used less often by the SD patients. The most frequently used long word for both groups was "Cinderella", which was used a total of 69 times by the controls and a total of 17 times by the SD patients (an average of 4.3 versus 1.7 times per narrative). Other long words that were also used more often by controls than patients include, for example, "slipper" which was used 48 times by controls but never by SD patients, and "beautiful" which was used 25 times by controls and 5 times by SD patients (an average of 1.6 versus 0.5 times per narrative). 
The final feature which distinguished well between the SD patients and controls was speech rate. As noted in the Introduction, findings with respect to speech rate in SD have been inconsistent. The slower rate for SD patients may be due to pauses in speech while searching for a word, and seems unlikely to reflect a motor speech problem. Wilson et al. (2010) also documented slower speech rate in SD patients than in controls, but found that the maximum speech rate for their patients was normal; they suggested that the slower rate reflected impairment in higher-level processes, and we concur with this idea.

The features that distinguish SD patients from controls can all be attributed to the semantic memory impairment. This seems to be the dominant influence in the language output of this group, and can account for the reliance on more frequent and familiar words, pauses for word-finding, and use of general terms such as demonstratives and adverbs like "then" and "so".

The features that distinguished the PNFA patients from controls were higher frequency of words and of verbs in particular, and reduced word length and speech rate. The feature that best distinguished PNFA from controls was word length. Increased word length of stimulus items has been shown to deleteriously affect naming, reading and repetition in PNFA (Croot et al., 1998; Graham et al., 2004), and this effect could arise from phonological or motor speech impairment(s). In the case of narrative speech, it could also arise from word finding difficulties, which would affect choice of words (as suggested for the SD patients). The current analyses do not inform the choice of explanation, and indeed the use of shorter words could arise from different causes in different individuals.

Not surprisingly, the PNFA patients had a slower rate of speech than controls, and this predicted group membership. A slower speech rate is one of the diagnostic features (GornoTempini et al., 2004), and has been documented in other studies of connected speech production in PNFA (Ash et al., 2010, 2006; Graham et al., 2004; Knibb et al., 2009; Thompson et al., 2012).

Like the SD patients, the PNFA patients were distinguished from controls by overall 
word frequency. For the PNFA patients (vs. controls), verb frequency also had a high selectivity ratio. Once again we have a situation where two correlated features both have high selectivity ratios, suggesting that each would have had an even higher ratio if they had been included in the model without the other. Studies have shown that naming of verbs/actions in PNFA is more impaired than naming of nouns/objects (Cotelli et al., 2006; Hillis et al., 2004). Although we do not know of a study which assesses the effect of frequency in naming of verbs in PNFA, our results suggest that production of higher frequency verbs is better preserved. The finding that the PNFA patients produce higher frequency verbs than the controls cannot be due to excessive use of light verbs (which tend to be high in frequency), because the $t$-tests indicate no difference between PNFA and controls in use of light verbs. Despite this apparent difficulty with verb production, the PNFA patients in this study produced nouns and verbs in normal proportions as demonstrated by equivalent noun-to-verb ratios for PNFA patients and controls. An increase in noun-to-verb ratio is taken to indicate difficulty with verb production, and has been reported in other studies of connected speech in PNFA (Thompson et al., 1997). Consistent with the current findings, Graham et al. (2004) also documented normal noun-to-verb ratios but suggested that the verbs produced by PNFA patients were less specific than those produced by controls.

The results also yielded interesting findings regarding the features that distinguished between the two patient groups. SD patients used more frequent nouns and less imageable words overall than PNFA patients. This is in keeping with the findings of previous research (Bird et al., 2000; Wilson et al., 2010; Thompson et al., 2012), which has shown that nouns are more affected than verbs in the connected speech of SD patients. Bird et al. suggested that the poorer performance with nouns was due to their lower overall frequency in comparison to verbs, or other word classes. The use of higher frequency nouns by the SD patients could be due to their greater semantic impairment, or to the fact that they are more anomic than the PNFA patients.

The other main feature that differentiated the two patient groups was imageability. SD 
patients produced words that were lower in imageability than PNFA patients. Although some have questioned whether SD patients show reverse imageability effects on a variety of tasks (see Jefferies et al. 2009), this tendency to produce words which are lower in imageability in connected speech has been found by others (e.g., Bird et al. 2000) and was most likely driven by the tendency to produce vague words (e.g., other, ever) by the SD patients, many of which, although high in frequency, are low in imageability. As with the differentiation from controls, the features that distinguish between SD patients and NFPA patients seem to be ones that reflect SD patients' semantic impairments.

Some surprising findings also emerged. While it is well documented that PNFA patients tend to have sparse output, producing fewer words than either controls or SD patients (Graham et al., 2004; Meteyard and Patterson, 2009; Wilson et al., 2010), there were no significant differences found on this measure between any of the groups nor did it emerge as an important feature distinguishing the groups. It may also be considered surprising that none of the features measuring syntactic complexity emerged as the main distinguishing features. As mentioned in Section 2.3, Lu's syntactic complexity analyzer was originally designed to be applied to written documents by second-language learners, and so we might not expect it to be ideally suited to the analysis of aphasic speech. Our goal was to test its performance in this domain, and although we found that it was effective in detecting clause boundaries, and returned counts that were highly correlated with manual counts, it did encounter difficulty in labelling types of clauses, and is limited by the fact that it does not output the structures themselves for further analysis. The adaptation of a similar tool to the domain of aphasic speech is a promising area of future research.

\subsection{Future directions}

In this work, we have demonstrated that fairly high classification accuracies can be achieved through automated quantitative analysis of speech samples, with relatively little human intervention required except for transcription. Given that procedures already exist for di- 
agnosing PPA (Gorno-Tempini et al., 2011), one might ask what added value there is in developing an automated classification approach based on naturalistic speech alone. In fact, a diagnostic classifier provides a starting point for a much more extensive use of speech samples in applications beyond diagnosis. The ultimate goal of research into language disorders is to develop techniques to intervene effectively, either to restore function, or to slow its decline. Many diagnostic tests are not generally well suited for longitudinal assessment of language function, due to practice and familiarity effects. Furthermore, a patients speech may change in significant ways that are not necessarily reflected by formal tests, but can be nonetheless captured and quantified by linguistic analysis. The automated measurement of many parameters provides the maximum opportunity to reveal significant changes across time, and the use of these parameters in classification provides a means to decide which parameters are most indicative of the function of underlying language systems.

We focused here on differential diagnosis between SD and PNFA, due to their association with degeneration in distinct brain regions. We consider SD and PNFA to be good models for studying dysfunction in the ventral and dorsal language networks, respectively. However, the same methods can be applied to tracking language dysfunction in a variety of neural disorders, including Alzheimer's Disease (AD). Language symptoms are relatively common in AD, but highly variable across individuals (Taler and Phillips, 2008). This high variability is presumably due to differential spread of cortical pathology (Stopford et al., 2008), in contrast to the medial temporal lobe pathology that is more universal in AD and underlies the diseases characteristic episodic memory impairment. As greater cortical involvement accompanies the progression of AD (Singh et al., 2006), quantitative analyses of speech content may provide a sensitive measure of disease severity, useful for the evaluation of interventions designed to slow the progression. Similarly, this analysis may be useful in contexts where the goal is to bring about improvement rather than slow decline, such as in rehabilitation of post-stroke aphasia. Although narrative speech is widely regarded as a rich and ecologically valid source of information about linguistic function, the labour-intensive 
nature of its analysis has precluded its widespread adoption in research and clinical practice. Therefore, the analysis methods presented here, which can be fully automated beyond the transcription stage, offer a basis for routine incorporation of narrative speech into cognitive evaluation for a wide range of disorders beyond PPA.

Longitudinal evaluation of language decline will require extensions to our present approach of binary classification. The binary classifiers presented here are aimed at categorizing an individual patient in an all-or-none fashion, and the metric of their success at present is the rate at which their classification matches the clinical diagnosis. However, some classifiers (e.g. naïve Bayes and logistic regression, out of the ones considered here) output a probabilistic estimate of class membership for each individual case. Such values could be tracked for an individual over time, based on a classifier trained on a static set of observations in different patients. Alternatively, instead of classification, one could use machine learning techniques based on regression, which seek to map continuous input variables to continuous output variables. Many such techniques are available, including ridge regression (Hoerl and Kennard, 1970), support vector regression, and relevance vector regression (Tipping, 2001). These techniques have become popular in neuroimaging, as investigators have sought to reveal relationships between continuous behavioural variables and multi-voxel measures of brain structure or activity (for review, see Cohen et al. (2011). In the case of PPA, these continuous coding techniques could be used to determine quantitative relationships between aspects of speech and patterns of brain atrophy or hypoactivity. As large datasets containing both speech data and neuroimaging measures from the same participants become available, we expect these machine learning methods to play an increasingly large role in elucidating the neural bases of language processing in both health and disease. 


\section{Acknowledgements}

This research was supported by a grant from the Canadian Institutes of Health Research (CIHR), grant \# MOP-82744 to E.R., S.E.B., C.L., and N.G. The authors also acknowledge the support of Toronto Rehabilitation Institute, which receives funding under the Provincial Rehabilitation Research Program from the Ministry of Health and Long-Term Care in Ontario. Additional support was provided by the Heart and Stroke Foundation Centre for

Stroke Recovery. We especially thank the participants for their patience and perseverance, and we thank Dr. David Tang-Wai and Dr. Tiffany Chow for referrals to the study. 


\section{References}

Ash S, McMillan C, Gunawardena D, Avants B, Morgan B, Khan A, Moore P, Gee J, and Grossman M. Speech errors in progressive non-fluent aphasia. Brain and Language, $113(1): 13-20,2010$.

Ash S, Moore P, Antani S, McCawley G, Work M, and Grossman M. Trying to tell a tale: Discourse impairments in progressive aphasia and frontotemporal dementia. Neurology, 66 (9):1405-1413, 2006.

Benedet M, Patterson K, Gomez-Pastor I, and Luisa Garcia de la Rocha M. 'Non-semantic' aspects of language in semantic dementia: As normal as they're said to be? Neurocase, $12(1): 15-26,2006$.

Berndt RS, Wayland S, Rochon E, Saffran E, and Schwartz M. Quantitative Production Analysis: A Training Manual for the Analysis of Aphasic Sentence Production. Hove, UK: Psychology Press, 2000.

Bird H, Lambon Ralph MA, Patterson K, and Hodges JR. The rise and fall of frequency and imageability: Noun and verb production in semantic dementia. Brain and Language, $73: 17-49,2000$.

Bishop DVM. Test for the Reception of Grammar (TROG-2) Version 2. London: Psychological Corporation, 2003.

Breedin SD, Saffran EM, and Schwartz MF. Semantic factors in verb retrieval: An effect of complexity. Brain and Language, 63:1-31, 1998.

Brysbaert M and New B. Moving beyond Kučera and Francis: A critical evaluation of current word frequency norms and the introduction of a new and improved word frequency measure for American English. Behavior Research Methods, 41 (4):977-990, 2009. 
Chen M and Zechner K. Computing and evaluating syntactic complexity features for automated scoring of spontaneous non-native speech. In Proceedings of the 49th Annual Meeting of the Association for Computational Linguistics: Human Language Technologies. Association for Computational Linguistics, 2011, HLT '11, 722-731.

Cheung $\mathrm{H}$ and Kemper S. Competing complexity metrics and adults' production of complex sentences. Applied Psycholinguistics, 13 (01):53-76, 1992.

Clark HH and Fox Tree JE. Using uh and um in spontaneous speaking. Cognition, 84 (1):73$111,2002$.

Cohen J, Asarnow R, Sabb F, Bilder R, Bookheimer S, Knowlton B, and Poldrack R. Decoding continuous variables from neuroimaging data: basic and clinical applications. Frontiers in Neuroscience, 5 (75), 2011.

Cotelli M, Borroni B, Manenti R, Alberici A, Calabria M, Agosti C, Arevalo A, Ginex V, Ortelli P, Binetti G, Zanetti O, Padovani A, and Cappa SF. Action and object naming in frontotemporal dementia, progressive supranuclear palsy, and corticobasal degeneration. Neuropsychology, 20 (5):558-565, 2006.

Croot K, Patterson K, and Hodges JR. Single word production in nonfluent progressive aphasia. Brain and Language, 61 (2):226-273, 1998.

Dunn LM and Dunn LM. Peabody Picture Vocabulary Test, third edition. Circle Pines, Minnesota: American Guidance Service, 1997.

Folstein MF, Folstein SE, and McHugh PR. "Mini-mental state". A practical method for grading the cognitive state of patients for the clinician. Journal of Psychiatric Research, 12:189-198, 1975.

Funnel E. W.L.P.: A case for the modularity of language function and dementia. In Code 
C, Wallesch CW, Joanette Y, and Lecours AR, eds., Classic Cases in Neuropsychology, Hove, UK: Psychology Press, 1996.

Garrard P and Forsyth R. Abnormal discourse in semantic dementia: a data-driven approach. Neurocase, 16 (6):520-528, 2010.

Gilhooly K and Logie R. Age-of-acquisition, imagery, concreteness, familiarity, and ambiguity measures for 1,944 words. Behavior Research Methods, 12:395-427, 1980.

Gorno-Tempini ML, Dronkers NF, Rankin KP, Ogar JM, Phengrasamy L, Rosen HJ, Johnson JK, Weiner MW, and Miller BL. Cognition and anatomy in three variants of primary progressive aphasia. Annals Neurol., 55 (3):335-346, 2004.

Gorno-Tempini ML, Hillis AE, Weintraub S, Kertesz A, Mendez M, Cappa SF, Ogar JM, Rohrer JD, Black S, Boeve BF, Manes F, Dronkers NF, Vandenberghe R, Rascovsky K, Patterson K, Miller BL, Knopman DS, Hodges JR, Mesulam MM, and Grossman M. Classification of primary progressive aphasia and its variants. Neurology, 76:1006-1014, 2011.

Graham NL, Patterson K, and Hodges JR. When more yields less: speaking and writing deficits in nonfluent progressive aphasia. Neurocase, 10 (2):141-155, 2004.

Haenlein M and Kaplan AM. A beginner's guide to partial least squares analysis. Understanding Statistics, 3 (4):283-297, 2004.

Hall M, Frank E, Holmes G, Pfahringer B, Reutmann P, and Witten IH. The WEKA data mining software: An update. ACM Special Interest Group on Knowledge Discovery and Data Mining Explorations, 2 (1), 2009.

Harciarek M and Kertesz A. Primary progressive aphasias and their contribution to the contemporary knowledge about the brain-language relationship. Neuropsychology Review, 21:271-287, 2011. 
Hillis AE, Oh S, and Ken L. Deterioration of naming nouns versus verbs in primary progressive aphasia. Annals of Neurology, 55 (2):268-275, 2004.

Hoerl AE and Kennard RW. Ridge regression: Biased estimation for nonorthogonal problems. Technometrics, 12 (1):55-67, 1970.

Jarrold W, Peintner B, Yeh E, Krasnow R, Javitz H, and Swan G. Language analytics for assessing brain health: Cognitive impairment, depression and pre-symptomatic Alzheimer's disease. In Yao Y, Sun R, Poggio T, Liu J, Zhong N, and Huang J, eds., Brain Informatics, Springer Berlin / Heidelberg, volume 6334 of Lecture Notes in Computer Science, 299-307, 2010.

Jefferies E, Patterson K, Jones RW, and Lambon Ralph MA. Comprehension of concrete and abstract words in semantic dementia. Neuropsychology, 23 (4):492-499, 2009.

Jurica PJ, Leitten CL, and Mattis S. Dementia Rating Scale-2. Lutz, FL: Psychological Assessment Resources, Inc., 2001.

Kaplan E, Goodglass H, and Weintraub S. Boston Naming Test, second edition. Philadelphia: Lippincott Williams \& Wilkins, 2001.

Kavé G, Leonard C, Cupit J, and Rochon E. Structurally well-formed narrative production in the face of severe conceptual deterioration: A longitudinal case study of a woman with semantic dementia. Journal of Neurolinguistics, 20 (2):161-177, 2007.

Klein D and Manning CD. Accurate unlexicalized parsing. In Proceedings of the 41st Meeting of the Association for Computational Linguistics, 2003, 423-430.

Knibb JA, Woollams AM, Hodges JR, and Patterson K. Making sense of progressive nonfluent aphasia: an analysis of conversational speech. Brain, 132 (10):2734-2746, 2009.

Kvalheim OM. Interpretation of partial least squares regression models by means of target projection and selectivity ratio plots. Journal of Chemometrics, 24 (7-8):496-504, 2010. 
Lambon Ralph MA, Graham KS, Ellis AW, and Hodges JR. Naming in semantic dementiawhat matters? Neuropsychologia, 36 (8):775-784, 1998.

Li H. Partial least squares-discriminant analysis and variable selection for high dimensional data (Matlab package), 2011.

Lu X. Automatic analysis of syntactic complexity in second language writing. International Journal of Corpus Linguistics, 15 (4):474-496, 2010.

Manning CD, Raghavan P, and Schütze H. Introduction to Information Retrieval. Cambridge University Press, 2008.

Manning CD and Schütze H. Foundations of Statistical Natural Language Processing. MIT Press, 1999.

Meteyard L and Patterson K. The relation between content and structure in language production: an analysis of speech errors in semantic dementia. Brain and Language, $110(3): 121-134,2009$.

Ng AY and Jordan MI. On discriminative vs. generative classifiers: A comparison of logistic regression and naive Bayes. In Advances in Neural Information Processing Systems (NIPS) 14, 2002, volume 2, 841-848.

Patterson K and MacDonald MC. Sweet nothings: Narrative speech in semantic dementia. In Andrews S, ed., From Inkmarks to Ideas: Current Issues in Lexical Processing, Hove, UK: Psychology Press, 2006.

Peintner B, Jarrold W, Vergyri D, Richey C, Tempini MLG, and Ogar J. Learning diagnostic models using speech and language measures. In Engineering in Medicine and Biology Society, 2008. EMBS 2008. 30th Annual International Conference of the IEEE, 2008, $4648-4651$.

Raven JC. Coloured Progressive Matrices Sets A, AB, B. London: H. K. Lewis, 1962. 
Rey A. L'examen psychologique dans les cas d'encephalopathie traumatique. Archives de Psychologie, 28:286-340, 1941.

Roark B, Mitchell M, Hosom JP, Hollingshead K, and Kaye J. Spoken language derived measures for detecting mild cognitive impairment. IEEE Transactions on Audio, Speech, and Language Processing, 19 (7):2081-2090, 2011.

Saffran EM, Berndt RS, and Schwartz MF. The quantitative analysis of agrammatic production: procedure and data. Brain and Language, 37:440-479, 1989.

Sajjadi SA, Patterson K, Tomek M, and Nestor PJ. Abnormalities of connected speech in semantic dementia vs Alzheimer's disease. Aphasiology, 26 (6):847-866, 2012.

Sampson G. Depth in English grammar. Journal of Linguistics, 33:131-51, 1997.

Simons JS, Graham KS, Galton CJ, Patterson K, and Hodges JR. Semantic knowledge and episodic memory for faces in semantic dementia. Neuropsychology, 15 (1):101-114, 2001.

Singh V, Chertkow H, Lerch JP, Evans AC, Dorr AE, and Kabani NJ. Spatial patterns of cortical thinning in mild cognitive impairment and Alzheimer's disease. Brain, 129 (11):28852893, 2006.

Stadthagen-Gonzalez H and Davis CJ. The Bristol norms for age of acquisition, imageability, and familiarity. Behavior Research Methods, 38 (4):598-605, 2006.

Stopford CL, Snowden JS, Thompson JC, and Neary D. Variability in cognitive presentation of Alzheimer's disease. Cortex, 44 (2):185-195, 2008.

Stromswold K, Caplan D, Alpert N, and Rauch S. Localization of syntactic comprehension by positron emission tomography. Brain Lang, 52 (3):452-473, 1996.

Taler V and Phillips NA. Language performance in Alzheimer's disease and mild cognitive impairment: a comparative review. Journal of Clinical and Experimental Psychology, $30(5): 501-556,2008$. 
Tanaka-Ishii K and Terada H. Word familiarity and frequency. Studia Linguistica, 96-116, 2011.

Thompson C, Ballard K, Tait M, Weintraub S, and Mesulam M. Patterns of language decline in non-fluent primary progressive aphasia. Aphasiology, 11:297-321, 1997.

Thompson CK, Cho S, Hsu CJ, Wieneke C, Rademaker A, Weitner BB, Mesulam MM, and Weintraub S. Dissociations between fluency and agrammatism in primary progressive aphasia. Aphasiology, 26 (1):20-43, 2012.

Tipping ME. Sparse Bayesian learning and the relevance vector machine. The Journal of Machine Learning Research, 1:211-244, 2001.

Toutanova K, Klein D, Manning C, and Singer Y. Feature-rich part-of-speech tagging with a cyclic dependency network. In Proceedings of the 2003 Conference of the North American Chapter of the Association for Computational Linguistics: Human Language Technologies, 2003, 252-259.

Warrington EK and James M. The Visual Object and Space Perception Battery. Bury St Edmunds: Thames Valley Test Company, 1991.

Wilson SM, Henry ML, Besbris M, Ogar JM, Dronkers NF, Jarrold W, Miller BL, and Gorno-Tempini ML. Connected speech production in three variants of primary progressive aphasia. Brain, 133:2069-2088, 2010.

Woollams AM, Cooper-Pye E, Hodges JR, and Patterson K. Anomia: a doubly typical signature of semantic dementia. Neuropsychologia, 46 (10):2503-2514, 2008.

Yngve V. A model and hypothesis for language structure. Proceedings of the American Physical Society, 104:444-466, 1960. 


\begin{tabular}{|c|c|c|c|c|}
\hline & $\begin{array}{l}\text { SD } \\
(n=10)\end{array}$ & $\begin{array}{l}\text { PNFA } \\
(n=14)\end{array}$ & $\begin{array}{l}\text { Controls } \\
(n=16)\end{array}$ & Group effect \\
\hline \multicolumn{5}{|l|}{ Demographic information } \\
\hline Age & $65.6(7.4)$ & $64.9(10.1)$ & $67.8(8.2)$ & ns \\
\hline Years of education & $17.5(6.1)$ & $14.3(3.6)$ & $16.8(4.3)$ & ns \\
\hline Sex & $3 \mathrm{~F}$ & $6 \mathrm{~F}$ & $7 \mathrm{~F}$ & \\
\hline Handedness & $9 \mathrm{R}$ & $13 \mathrm{R}$ & $16 \mathrm{R}$ & \\
\hline \multicolumn{5}{|l|}{ General cognitive function } \\
\hline Mini-Mental State Examination $(/ 30)$ & $24.4(4.3)^{a}$ & $25.0(2.9)^{a}$ & $29.3(0.8)$ & $* * *$ \\
\hline Dementia Rating Scale (/144) & $117.2(12.6)^{a}$ & $123.9(15.6)^{a}$ & $142.2(1.7)$ & $* * *$ \\
\hline \multicolumn{5}{|l|}{ Language production } \\
\hline Boston Naming $(/ 60)$ & $13.9(7.3)^{a, b}$ & $39.6(11.5)^{a}$ & $55.8(3.3)$ & $* * *$ \\
\hline Category fluency — animals & $7.6(3.7)^{a}$ & $12.3(6.0)^{a}$ & $20.4(4.4)$ & $* * *$ \\
\hline \multicolumn{5}{|l|}{ Language comprehension } \\
\hline TROG $(/ 80)$ & $71.4(11.0)$ & $63.9(12.0)^{a}$ & $79.1(0.9)$ & $* * *$ \\
\hline Peabody Picture Vocabulary Test (/204) & $113.8(30.8)^{a, b}$ & $172.9(14.3)^{a}$ & $196.1(3.9)$ & $* * *$ \\
\hline \multicolumn{5}{|l|}{ Visuospatial } \\
\hline Copy of Rey Complex Figure (/36) & $33.2(2.6)$ & $29.9(5.3)^{a}$ & $33.4(1.4)$ & * \\
\hline VOSP cube analysis subtest $(/ 10)$ & $9.4(1.9)$ & $9.2(1.6)$ & $8.5(2.1)$ & ns \\
\hline \multicolumn{5}{|l|}{ Nonverbal memory } \\
\hline 30 minute recall of Rey Complex Figure $(/ 36)$ & $12.7(7.1)$ & $14.9(6.3)$ & $18.8(6.9)$ & ns \\
\hline \multicolumn{5}{|l|}{ Nonverbal reasoning } \\
\hline Raven's Coloured Progressive Matrices (/36) & $31.5(5.0)$ & $27.1(6.5)^{a}$ & $31.8(4.2)$ & * \\
\hline
\end{tabular}

Table 1: Demographic and neuropsychological data for each participant group. Values shown are mean (SD). Asterisks denote significant effect of group on 1-way analyses of variance at $* p<.05, * * * \quad p<.001$.

${ }^{a}$ Significantly different from controls

${ }^{b}$ Significantly different from nonfluent patients 
Table 2: Definitions of features.

\begin{tabular}{|c|c|c|}
\hline & Feature & Definition \\
\hline 1 & Words & \# words \\
\hline 2 & Sentences & \# sentences \\
\hline 3 & T-units & a clause and all of its dependent clauses \\
\hline 4 & Clauses & a structure consisting of at least a subject and a finite verb \\
\hline 5 & Coordinate phrases & a phrase immediately before a coordinating conjunction \\
\hline 6 & Complex nominals & a noun phrase, clause, or gerund that stands in for a noun \\
\hline 7 & Complex T-units & a T-unit which contains a dependent clause \\
\hline 8 & Verb phrases & a phrase consisting of at least a verb and its dependents \\
\hline 9 & Dependent clauses & a clause which could not form a sentence on its own \\
\hline 10 & Mean length of sentence & mean length of sentence \\
\hline 11 & Mean length of clause & mean length of clause \\
\hline 12 & Mean length of T-unit & mean length of T-unit \\
\hline 13 & Dependent clauses per clause & \\
\hline 14 & Dependent clauses per T-unit & \\
\hline 15 & Verb phrases er T-unit & \\
\hline 16 & Clauses per sentence & \\
\hline 17 & Clauses per T-unit & \\
\hline 18 & Complex T-units per T-unit & \\
\hline 19 & Coordinate phrases per T-unit & \\
\hline 20 & Complex nominals per T-unit & \\
\hline 21 & T-units per sentence & \\
\hline 22 & Coordinate phrases per clause & \\
\hline 23 & Complex nominals per clause & \\
\hline 24 & Tree height & height of the parse tree \\
\hline 25 & Total depth & total Yngve depth \\
\hline 26 & Max depth & maximum Yngve depth \\
\hline 27 & Mean depth & mean Yngve depth \\
\hline 28 & Nouns & \# nouns / \# words \\
\hline 29 & Verbs & \# verbs / \# words \\
\hline 30 & Noun-verb ratio & \# nouns / \# verbs \\
\hline 31 & Noun ratio & \# nouns / (\# nouns + \# verbs) \\
\hline 32 & Inflected verbs & \# inflected verbs / \# verbs \\
\hline 33 & Light verbs & \# light verbs / \# verbs \\
\hline 34 & Determiners & \# determiners / \# words \\
\hline 35 & Demonstratives & \# demonstratives / \# words \\
\hline 36 & Prepositions & \# prepositions / \# words \\
\hline 37 & Adjectives & \# adjectives / \# words \\
\hline 38 & Adverbs & \# adverbs / \# words \\
\hline 39 & Pronoun ratio & \# pronouns / (\# nouns + \# pronouns) \\
\hline 40 & Function words & \# function words / \# words \\
\hline 41 & Frequency & mean frequency of all words appearing in the frequency norms \\
\hline 42 & Noun frequency & mean frequency of nouns appearing in the frequency norms \\
\hline 43 & Verb frequency & mean frequency of verbs appearing in the frequency norms \\
\hline 44 & Imageability & mean imageability of all words appearing in the imageability norms \\
\hline 45 & Noun imageability & mean imageability of nouns appearing in the imageability norms \\
\hline 46 & Verb imageability & mean imageability of verbs appearing in the imageability norms \\
\hline 47 & Age of acquisition & mean age of acquisition of all words appearing in the age of acquisition norms \\
\hline 48 & Noun age of acquisition & mean age of acquisition of nouns appearing in the age of acquisition norms \\
\hline 49 & Verb age of acquisition & mean age of acquisition of verbs appearing in the age of acquisition norms \\
\hline 50 & Familiarity & mean familiarity of all words appearing in the familiarity norms \\
\hline 51 & Noun familiarity & mean familiarity of nouns appearing in the familiarity norms \\
\hline 52 & Verb familiarity & mean familiarity of verbs appearing in the familiarity norms \\
\hline 53 & Type-token ratio & type-token ratio: \# unique word types / \# words \\
\hline 54 & Word length & mean number of letters in each word \\
\hline 55 & Fillers & \# fillers / \# words \\
\hline 56 & Um & \# occurrences of 'um' / \# words \\
\hline 57 & Uh & \# occurrences of 'uh' / \# words \\
\hline 58 & Speech rate & \# words uttered / total time in minutes \\
\hline
\end{tabular}


Table 3: Correlations between human- and computer-generated counts for syntactic structures.

\begin{tabular}{|l|l|l|l|l|}
\hline Clauses & Dependent clauses & T-units & Complex T-units & Coordinate phrases \\
\hline 0.9966 & 0.9319 & 0.9269 & 0.8792 & 0.9475 \\
\hline
\end{tabular}


Table 4: A comparison of SD and control features. Values shown are mean (standard deviation). Asterisks denote significance $\left(* p<.05 ;{ }^{* *} p<.01\right)$.

\begin{tabular}{|c|c|c|c|c|c|}
\hline \multicolumn{2}{|c|}{ Feature } & \multirow{2}{*}{$\begin{array}{l}\text { SD } \\
380.300(272.429)\end{array}$} & \multirow{2}{*}{$\begin{array}{l}\text { Controls } \\
403.688(121.380)\end{array}$} & \multirow{2}{*}{\multicolumn{2}{|c|}{$\begin{array}{l}p \text { value } \\
0.8025\end{array}$}} \\
\hline 1 & Words & & & & \\
\hline 2 & Sentences & $20.400(13.550)$ & $15.688(6.877)$ & 0.3276 & \\
\hline 3 & T-units & $0.069(0.022)$ & $0.058(0.019)$ & 0.2036 & \\
\hline 4 & Clauses & $0.145(0.013)$ & $0.133(0.011)$ & 0.0292 & * \\
\hline 5 & Coordinate phrases & $0.028(0.010)$ & $0.029(0.007)$ & 0.6676 & \\
\hline 6 & Complex nominals & $0.098(0.039)$ & $0.083(0.020)$ & 0.2735 & \\
\hline 7 & Complex T-units & $0.037(0.009)$ & $0.030(0.009)$ & 0.0505 & \\
\hline 8 & Verb phrases & $0.177(0.020)$ & $0.167(0.015)$ & 0.2056 & \\
\hline 9 & Dependent clauses & $0.067(0.016)$ & $0.052(0.021)$ & 0.0543 & \\
\hline 10 & Mean length of sentence & $20.135(8.190)$ & $28.608(10.973)$ & 0.0346 & * \\
\hline 11 & Mean length of clause & $7.035(0.603)$ & $7.602(0.632)$ & 0.0329 & * \\
\hline 12 & Mean length of T-unit & $16.217(6.481)$ & $19.465(8.042)$ & 0.2701 & \\
\hline 13 & Dependent clauses per clause & $0.460(0.090)$ & $0.391(0.144)$ & 0.1459 & \\
\hline 14 & Dependent clauses per T-unit & $1.094(0.497)$ & $1.081(0.768)$ & 0.9571 & \\
\hline 15 & Verb phrases per T-unit & $2.848(1.276)$ & $3.186(1.196)$ & 0.5094 & \\
\hline 16 & Clauses per sentence & $2.845(1.010)$ & $3.738(1.242)$ & 0.0573 & \\
\hline 17 & Clauses per T-unit & $2.292(0.786)$ & $2.558(1.007)$ & 0.4610 & \\
\hline 18 & Complex T-units per T-unit & $0.580(0.183)$ & $0.535(0.159)$ & 0.5353 & \\
\hline 19 & Coordinate phrases per T-unit & $0.463(0.334)$ & $0.589(0.369)$ & 0.3820 & \\
\hline 20 & Complex nominals per $\mathrm{T}$-unit & $1.558(0.720)$ & $1.694(1.082)$ & 0.7038 & \\
\hline 21 & T-units per Sentence & $1.250(0.230)$ & $1.526(0.356)$ & 0.0249 & * \\
\hline 22 & Coordinate phrases per clause & $0.193(0.074)$ & $0.221(0.058)$ & 0.3150 & \\
\hline 23 & Complex nominals per clause & $0.673(0.229)$ & $0.627(0.162)$ & 0.5911 & \\
\hline 24 & Tree height & $13.167(2.359)$ & $14.821(2.401)$ & 0.0998 & \\
\hline 25 & Total Yngve depth & $70.477(41.393)$ & $117.609(72.438)$ & 0.0456 & * \\
\hline 26 & Maximum Yngve depth & $5.091(1.168)$ & $6.023(1.156)$ & 0.0613 & \\
\hline 27 & Mean Yngve Depth & $2.913(0.409)$ & $3.345(0.498)$ & 0.0250 & * \\
\hline 28 & Nouns & $0.141(0.031)$ & $0.179(0.026)$ & 0.0051 & $* *$ \\
\hline 29 & Verbs & $0.207(0.028)$ & $0.200(0.019)$ & 0.4427 & \\
\hline 30 & Noun-verb ratio & $0.699(0.209)$ & $0.907(0.163)$ & 0.0164 & * \\
\hline 31 & Noun ratio & $0.403(0.072)$ & $0.472(0.047)$ & 0.0178 & * \\
\hline 32 & Inflected verbs & $0.635(0.127)$ & $0.706(0.086)$ & 0.1417 & \\
\hline 33 & Light verbs & $0.474(0.093)$ & $0.476(0.085)$ & 0.9527 & \\
\hline 34 & Determiners & $0.107(0.030)$ & $0.120(0.016)$ & 0.2203 & \\
\hline 35 & Demonstratives & $0.037(0.011)$ & $0.012(0.009)$ & 0.0000 & ** \\
\hline 36 & Prepositions & $0.103(0.035)$ & $0.087(0.015)$ & 0.1994 & \\
\hline 37 & Adjectives & $0.034(0.013)$ & $0.038(0.009)$ & 0.3434 & \\
\hline 38 & Adverbs & $0.083(0.017)$ & $0.058(0.014)$ & 0.0010 & ** \\
\hline 39 & Pronoun ratio & $0.508(0.094)$ & $0.416(0.068)$ & 0.0175 & * \\
\hline 40 & Function words & $0.467(0.033)$ & $0.453(0.033)$ & 0.2823 & \\
\hline 41 & Frequency & $5.021(0.105)$ & $4.803(0.104)$ & 0.0001 & $* *$ \\
\hline 42 & Noun frequency & $3.861(0.231)$ & $3.282(0.183)$ & 0.0000 & ** \\
\hline 43 & Verb frequency & $4.614(0.282)$ & $4.378(0.184)$ & 0.0341 & * \\
\hline 44 & Imageability & $477.721(44.025)$ & $507.025(20.643)$ & 0.0729 & \\
\hline 45 & Noun imageability & $560.959(43.450)$ & $580.710(12.370)$ & 0.1913 & \\
\hline 46 & Verb imageability & $416.117(37.506)$ & $385.947(22.543)$ & 0.0387 & * \\
\hline 47 & Age of acquisition & $258.881(21.629)$ & $257.814(12.476)$ & 0.8894 & \\
\hline 48 & Noun age of acquisition & $254.246(33.465)$ & $251.696(19.006)$ & 0.8295 & \\
\hline 49 & Verb age of acquisition & $260.465(27.603)$ & $266.521(14.566)$ & 0.5338 & \\
\hline 50 & Familiarity & $607.358(12.903)$ & $565.956(10.052)$ & 0.0000 & ** \\
\hline 51 & Noun familiarity & $604.910(20.954)$ & 545.967 (17.119) & 0.0000 & ** \\
\hline 52 & Verb familiarity & $605.526(19.573)$ & $600.218(13.388)$ & 0.4629 & \\
\hline 53 & Type-token ratio & $0.405(0.118)$ & $0.415(0.057)$ & 0.8028 & \\
\hline 54 & Mean word length & $3.735(0.186)$ & $3.997(0.152)$ & 0.0017 & $* *$ \\
\hline 55 & Fillers & $0.053(0.067)$ & $0.054(0.056)$ & 0.9876 & \\
\hline 56 & Um & $0.007(0.008)$ & $0.014(0.015)$ & 0.1613 & \\
\hline 57 & Uh & $0.046(0.061)$ & $0.040(0.060)$ & 0.8052 & \\
\hline 58 & Speech rate & $104.048(35.149)$ & $160.779(35.131)$ & 0.0007 & ** \\
\hline
\end{tabular}


Table 5: A comparison of PNFA and control features. Values shown are mean (standard deviation). Asterisks denote significance $\left(* p<.05 ;{ }^{* *} p<.01\right)$.

\begin{tabular}{|c|c|c|c|c|c|}
\hline \multicolumn{2}{|c|}{ Feature } & \multirow{2}{*}{$\begin{array}{l}\text { PNFA } \\
302.214(141.837)\end{array}$} & \multirow{2}{*}{$\begin{array}{l}\text { Controls } \\
403.688(121.380)\end{array}$} & \multirow{2}{*}{$\begin{array}{l}p \text { value } \\
0.0466\end{array}$} & \multirow[b]{2}{*}{ * } \\
\hline 1 & Words & & & & \\
\hline 2 & Sentences & $16.143(12.526)$ & $15.688(6.877)$ & 0.9049 & \\
\hline 3 & T-units & $0.061(0.023)$ & $0.058(0.019)$ & 0.7698 & \\
\hline 4 & Clauses & $0.141(0.020)$ & $0.133(0.011)$ & 0.2027 & \\
\hline 5 & Coordinate phrases & $0.028(0.013)$ & $0.029(0.007)$ & 0.7585 & \\
\hline 6 & Complex nominals & $0.092(0.017)$ & $0.083(0.020)$ & 0.2005 & \\
\hline 7 & Complex T-units & $0.030(0.010)$ & $0.030(0.009)$ & 0.9345 & \\
\hline 8 & Verb phrases & $0.167(0.017)$ & $0.167(0.015)$ & 0.9699 & \\
\hline 9 & Dependent clauses & $0.055(0.016)$ & $0.052(0.021)$ & 0.7312 & \\
\hline 10 & Mean length of sentence & $24.501(12.192)$ & $28.608(10.973)$ & 0.3437 & \\
\hline 11 & Mean length of clause & $7.299(0.986)$ & $7.602(0.632)$ & 0.3348 & \\
\hline 12 & Mean length of T-unit & $19.655(8.814)$ & $19.465(8.042)$ & 0.9516 & \\
\hline 13 & Dependent clauses per clause & $0.384(0.082)$ & $0.391(0.144)$ & 0.1333 & \\
\hline 14 & Dependent clauses per T-unit & $1.054(0.545)$ & $1.081(0.768)$ & 0.9106 & \\
\hline 15 & Verb phrases per T-unit & $3.212(1.400)$ & $3.186(1.196)$ & 0.9577 & \\
\hline 16 & Clauses per sentence & $3.346(1.493)$ & $3.738(1.242)$ & 0.4444 & \\
\hline 17 & Clauses per T-unit & $2.716(1.243)$ & $2.558(1.007)$ & 0.7078 & \\
\hline 18 & Complex T-units per T-unit & $0.517(0.146)$ & $0.535(0.159)$ & 0.7456 & \\
\hline 19 & Coordinate phrases per T-unit & $0.615(0.521)$ & $0.589(0.369)$ & 0.8763 & \\
\hline 20 & Complex nominals per $\mathrm{T}$-unit & $1.767(0.790)$ & $1.694(1.082)$ & 0.8331 & \\
\hline 21 & T-units per Sentence & $1.256(0.254)$ & $1.526(0.356)$ & 0.0232 & $*$ \\
\hline 22 & Coordinate phrases per clause & $0.203(0.093)$ & $0.221(0.058)$ & 0.5336 & \\
\hline 23 & Complex nominals per clause & $0.660(0.130)$ & $0.627(0.162)$ & 0.5493 & \\
\hline 24 & Tree height & $12.933(2.718)$ & $14.821(2.401)$ & 0.0555 & \\
\hline 25 & Total Yngve depth & $108.405(78.010)$ & $117.609(72.438)$ & 0.7415 & \\
\hline 26 & Maximum Yngve depth & $5.275(1.364)$ & $6.023(1.156)$ & 0.1197 & \\
\hline 27 & Mean Yngve Depth & $3.085(0.589)$ & $3.345(0.498)$ & 0.2070 & \\
\hline 28 & Nouns & $0.155(0.040)$ & $0.179(0.026)$ & 0.0644 & \\
\hline 29 & Verbs & $0.191(0.025)$ & $0.200(0.019)$ & 0.2804 & \\
\hline 30 & Noun-verb ratio & $0.837(0.286)$ & $0.907(0.163)$ & 0.4276 & \\
\hline 31 & Noun ratio & $0.444(0.082)$ & $0.472(0.047)$ & 0.2775 & \\
\hline 32 & Inflected verbs & $0.706(0.096)$ & $0.706(0.086)$ & 0.9923 & \\
\hline 33 & Light verbs & $0.538(0.141)$ & $0.476(0.085)$ & 0.1666 & \\
\hline 34 & Determiners & $0.130(0.032)$ & $0.120(0.016)$ & 0.3183 & \\
\hline 35 & Demonstratives & $0.026(0.018)$ & $0.012(0.009)$ & 0.0210 & $*$ \\
\hline 36 & Prepositions & $0.088(0.032)$ & $0.087(0.015)$ & 0.8884 & \\
\hline 37 & Adjectives & $0.030(0.017)$ & $0.038(0.009)$ & 0.1219 & \\
\hline 38 & Adverbs & $0.069(0.030)$ & $0.058(0.014)$ & 0.2253 & \\
\hline 39 & Pronoun ratio & $0.476(0.095)$ & $0.416(0.068)$ & 0.0601 & \\
\hline 40 & Function words & $0.478(0.045)$ & $0.453(0.033)$ & 0.0968 & \\
\hline 41 & Frequency & $4.962(0.118)$ & $4.803(0.104)$ & 0.0006 & $* *$ \\
\hline 42 & Noun frequency & $3.451(0.317)$ & $3.282(0.183)$ & 0.0936 & \\
\hline 43 & Verb frequency & $4.608(0.237)$ & $4.378(0.184)$ & 0.0072 & ** \\
\hline 44 & Imageability & $509.119(40.552)$ & $507.025(20.643)$ & 0.8634 & \\
\hline 45 & Noun imageability & $579.078(23.669)$ & $580.710(12.370)$ & 0.8192 & \\
\hline 46 & Verb imageability & $404.073(49.196)$ & $385.947(22.543)$ & 0.2215 & \\
\hline 47 & Age of acquisition & $245.879(14.862)$ & $257.814(12.476)$ & 0.0260 & $*$ \\
\hline 48 & Noun age of acquisition & $235.038(20.937)$ & $251.696(19.006)$ & 0.0316 & $*$ \\
\hline 49 & Verb age of acquisition & $264.400(13.607)$ & $266.521(14.566)$ & 0.6834 & \\
\hline 50 & Familiarity & $573.625(18.408)$ & $565.956(10.052)$ & 0.1808 & \\
\hline 51 & Noun familiarity & $561.110(24.689)$ & 545.967 (17.119) & 0.0668 & \\
\hline 52 & Verb familiarity & $589.838(19.242)$ & $600.218(13.388)$ & 0.1043 & \\
\hline 53 & Type-token ratio & $0.421(0.046)$ & $0.415(0.057)$ & 0.7421 & \\
\hline 54 & Mean word length & $3.769(0.136)$ & $3.997(0.152)$ & 0.0002 & $* *$ \\
\hline 55 & Fillers & $0.083(0.080)$ & $0.054(0.056)$ & 0.2584 & \\
\hline 56 & Um & $0.025(0.027)$ & $0.014(0.015)$ & 0.1948 & \\
\hline 57 & Uh & $0.058(0.084)$ & $0.040(0.060)$ & 0.5937 & \\
\hline 58 & Speech rate & $78.468(27.978)$ & $160.779(35.131)$ & 0.0000 & $* *$ \\
\hline
\end{tabular}


Table 6: A comparison of SD and PNFA features. Values shown are mean (standard deviation). Asterisks denote significance $\left({ }^{*} p<.05 ; * * p<.01\right)$.

\begin{tabular}{|c|c|c|c|c|c|}
\hline \multicolumn{2}{|c|}{ Feature } & \multirow{2}{*}{$\begin{array}{l}\text { SD } \\
380.300(272.429)\end{array}$} & \multirow{2}{*}{$\begin{array}{l}\text { PNFA } \\
302.214(141.837)\end{array}$} & \multirow{2}{*}{$\begin{array}{l}p \text { value } \\
0.4223\end{array}$} & \\
\hline 1 & Words & & & & \\
\hline 2 & Sentences & $20.400(13.550)$ & $16.143(12.526)$ & 0.4436 & \\
\hline 3 & T-units & $0.069(0.022)$ & $0.061(0.023)$ & 0.3518 & \\
\hline 4 & Clauses & $0.145(0.013)$ & $0.141(0.020)$ & 0.6028 & \\
\hline 5 & Coordinate phrases & $0.028(0.010)$ & $0.028(0.013)$ & 0.9334 & \\
\hline 6 & Complex nominals & $0.098(0.039)$ & $0.092(0.017)$ & 0.6376 & \\
\hline 7 & Complex T-units & $0.037(0.009)$ & $0.030(0.010)$ & 0.0580 & \\
\hline 8 & Verb phrases & $0.177(0.020)$ & $0.167(0.017)$ & 0.2393 & \\
\hline 9 & Dependent clauses & $0.067(0.016)$ & $0.055(0.016)$ & 0.0805 & \\
\hline 10 & Mean length of sentence & $20.135(8.190)$ & $24.501(12.192)$ & 0.3056 & \\
\hline 11 & Mean length of clause & $7.035(0.603)$ & $7.299(0.986)$ & 0.4254 & \\
\hline 12 & Mean length of T-unit & $16.217(6.481)$ & $19.655(8.814)$ & 0.2829 & \\
\hline 13 & Dependent clauses per clause & $0.460(0.090)$ & $0.384(0.082)$ & 0.0472 & * \\
\hline 14 & Dependent clauses per T-unit & $1.094(0.497)$ & $1.054(0.545)$ & 0.8509 & \\
\hline 15 & Verb phrases per T-unit & $2.848(1.276)$ & $3.212(1.400)$ & 0.5161 & \\
\hline 16 & Clauses per sentence & $2.845(1.010)$ & $3.346(1.493)$ & 0.3381 & \\
\hline 17 & Clauses per T-unit & $2.292(0.786)$ & $2.716(1.243)$ & 0.3188 & \\
\hline 18 & Complex T-units per T-unit & $0.580(0.183)$ & $0.517(0.146)$ & 0.3825 & \\
\hline 19 & Coordinate phrases per $\mathrm{T}$-unit & $0.463(0.334)$ & $0.615(0.521)$ & 0.3953 & \\
\hline 20 & Complex nominals per $\mathrm{T}$-unit & $1.558(0.720)$ & $1.767(0.790)$ & 0.5084 & \\
\hline 21 & T-units per Sentence & $1.250(0.230)$ & $1.256(0.254)$ & 0.9541 & \\
\hline 22 & Coordinate phrases per clause & $0.193(0.074)$ & $0.203(0.093)$ & 0.7648 & \\
\hline 23 & Complex nominals per clause & $0.673(0.229)$ & $0.660(0.130)$ & 0.8714 & \\
\hline 24 & Tree height & $13.167(2.359)$ & $12.933(2.718)$ & 0.8246 & \\
\hline 25 & Total Yngve depth & $70.477(41.393)$ & $108.405(78.010)$ & 0.1386 & \\
\hline 26 & Maximum Yngve depth & $5.091(1.168)$ & $5.275(1.364)$ & 0.7270 & \\
\hline 27 & Mean Yngve Depth & $2.913(0.409)$ & $3.085(0.589)$ & 0.4071 & \\
\hline 28 & Nouns & $0.141(0.031)$ & $0.155(0.040)$ & 0.3591 & \\
\hline 29 & Verbs & $0.207(0.028)$ & $0.191(0.025)$ & 0.1440 & \\
\hline 30 & Noun-verb ratio & $0.699(0.209)$ & $0.837(0.286)$ & 0.1844 & \\
\hline 31 & Noun ratio & $0.403(0.072)$ & $0.444(0.082)$ & 0.2122 & \\
\hline 32 & Inflected verbs & $0.635(0.127)$ & $0.706(0.096)$ & 0.1550 & \\
\hline 33 & Light verbs & $0.474(0.093)$ & $0.538(0.141)$ & 0.1935 & \\
\hline 34 & Determiners & $0.107(0.030)$ & $0.130(0.032)$ & 0.0862 & \\
\hline 35 & Demonstratives & $0.037(0.011)$ & $0.026(0.018)$ & 0.0688 & \\
\hline 36 & Prepositions & $0.103(0.035)$ & $0.088(0.032)$ & 0.3086 & \\
\hline 37 & Adjectives & $0.034(0.013)$ & $0.030(0.017)$ & 0.5577 & \\
\hline 38 & Adverbs & $0.083(0.017)$ & $0.069(0.030)$ & 0.1586 & \\
\hline 39 & Pronoun ratio & $0.508(0.094)$ & $0.476(0.095)$ & 0.4313 & \\
\hline 40 & Function words & $0.467(0.033)$ & $0.478(0.045)$ & 0.5254 & \\
\hline 41 & Frequency & $5.021(0.105)$ & $4.962(0.118)$ & 0.2139 & \\
\hline 42 & Noun frequency & $3.861(0.231)$ & $3.451(0.317)$ & 0.0014 & 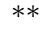 \\
\hline 43 & Verb frequency & $4.614(0.282)$ & $4.608(0.237)$ & 0.9557 & \\
\hline 44 & Imageability & $477.721(44.025)$ & $509.119(40.552)$ & 0.0916 & \\
\hline 45 & Noun imageability & $560.959(43.450)$ & $579.078(23.669)$ & 0.2527 & \\
\hline 46 & Verb imageability & $416.117(37.506)$ & $404.073(49.196)$ & 0.5036 & \\
\hline 47 & Age of acquisition & $258.881(21.629)$ & $245.879(14.862)$ & 0.1211 & \\
\hline 48 & Noun age of acquisition & $254.246(33.465)$ & $235.038(20.937)$ & 0.1309 & \\
\hline 49 & Verb age of acquisition & $260.465(27.603)$ & $264.400(13.607)$ & 0.6845 & \\
\hline 50 & Familiarity & $607.358(12.903)$ & $573.625(18.408)$ & 0.0000 & $* *$ \\
\hline 51 & Noun familiarity & $604.910(20.954)$ & $561.110(24.689)$ & 0.0001 & $* *$ \\
\hline 52 & Verb familiarity & $605.526(19.573)$ & $589.838(19.242)$ & 0.0659 & \\
\hline 53 & Type-token ratio & $0.405(0.118)$ & $0.421(0.046)$ & 0.6828 & \\
\hline 54 & Mean word length & $3.735(0.186)$ & $3.769(0.136)$ & 0.6341 & \\
\hline 55 & Fillers & $0.053(0.067)$ & $0.083(0.080)$ & 0.3290 & \\
\hline 56 & Um & $0.007(0.008)$ & $0.025(0.027)$ & 0.0335 & * \\
\hline 57 & Uh & $0.046(0.061)$ & $0.058(0.084)$ & 0.6864 & \\
\hline 58 & Speech rate & $104.048(35.149)$ & $78.468(27.978)$ & 0.0736 & \\
\hline
\end{tabular}


Table 7: Accuracies for the three classifiers, compared to a simple baseline classifier.

\begin{tabular}{|l|c|c|c|}
\hline & SD vs. control & PNFA vs. control & SD vs. PNFA \\
\hline Baseline & .615 & .533 & .583 \\
Naïve Bayes & .923 & .900 & .792 \\
Logistic Regression & .962 & .933 & .708 \\
SVM & 1.00 & .967 & .750 \\
\hline
\end{tabular}


Figure 1: Partial least squares analysis of the data, with each point representing one transcript.

(a) SD vs control

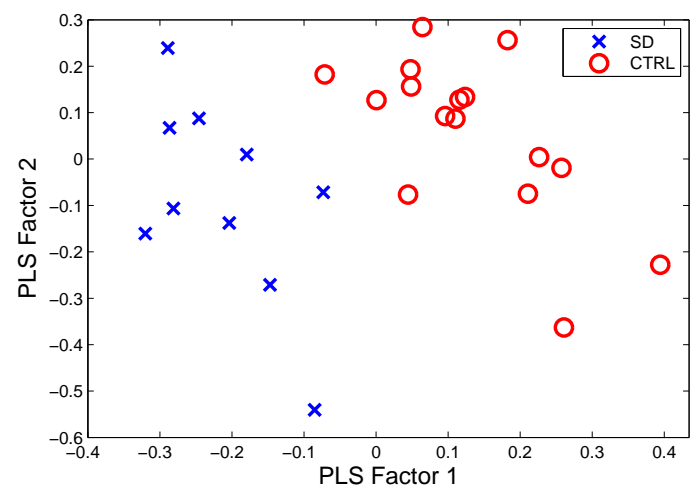

(b) PNFA vs control

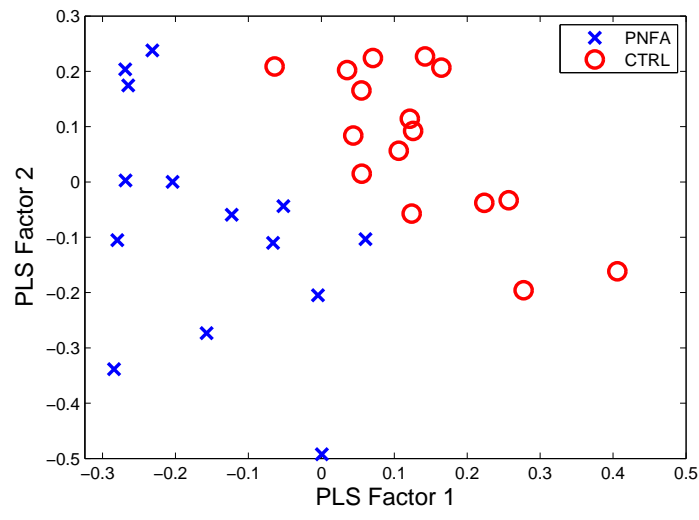

(c) SD vs PNFA

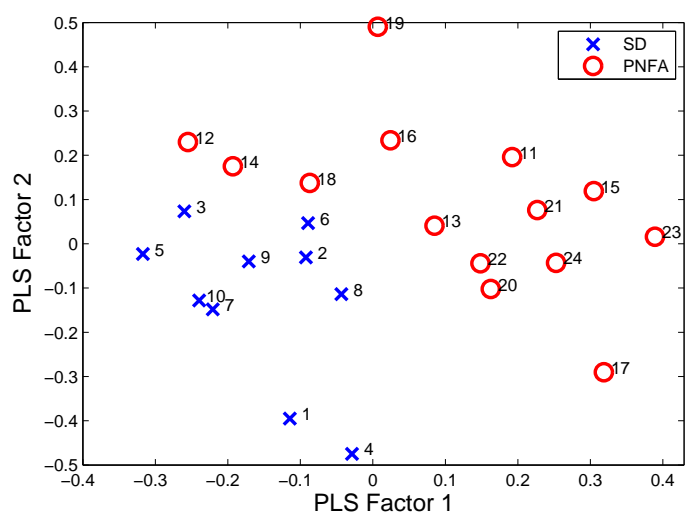


Figure 2: PLS selectivity ratio for each of the features. On the left, plots of the selectivity ratio for every feature. The feature numbers on the horizontal axes refer to the feature numbers in Table 2. Note the difference in scale on the vertical axes. On the right, the features with selectivity ratio greater than 0.5 .

(a) SD vs. control

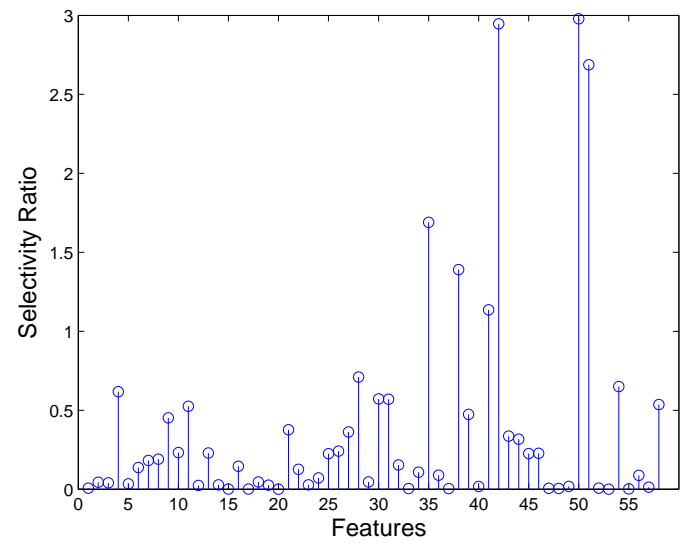

\begin{tabular}{|l|l|l|}
\hline \multicolumn{2}{|l|}{ Feature } & SR \\
\hline 50 & familiarity & 2.98 \\
42 & noun frequency & 2.95 \\
51 & noun familiarity & 2.69 \\
35 & demonstratives & 1.69 \\
38 & adverbs & 1.39 \\
41 & frequency & 1.13 \\
28 & nouns & 0.71 \\
54 & word length & 0.65 \\
4 & clauses & 0.62 \\
30 & noun-verb ratio & 0.57 \\
31 & noun ratio & 0.57 \\
58 & speech rate & 0.54 \\
11 & mean clause length & 0.53 \\
\hline
\end{tabular}

(b) PNFA vs. control

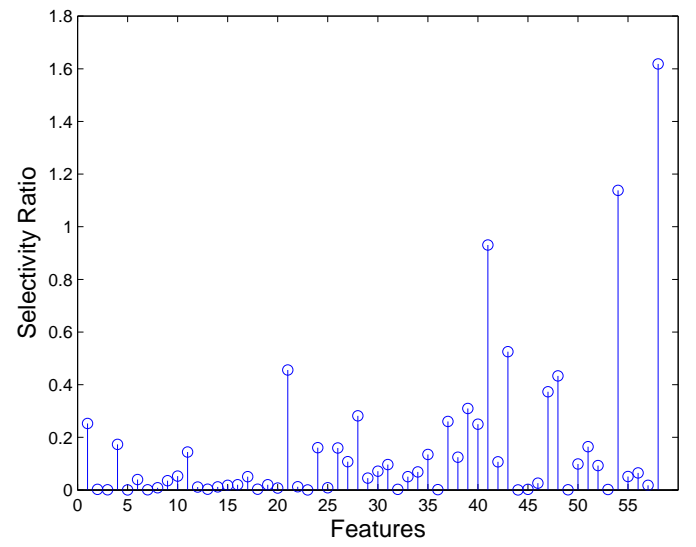

\begin{tabular}{|l|l|l|}
\hline \multicolumn{2}{|l|}{ Feature } & SR \\
\hline 58 & speech rate & 1.62 \\
54 & word length & 1.14 \\
41 & frequency & 0.93 \\
43 & verb frequency & 0.53 \\
\hline
\end{tabular}

(c) SD vs. PNFA

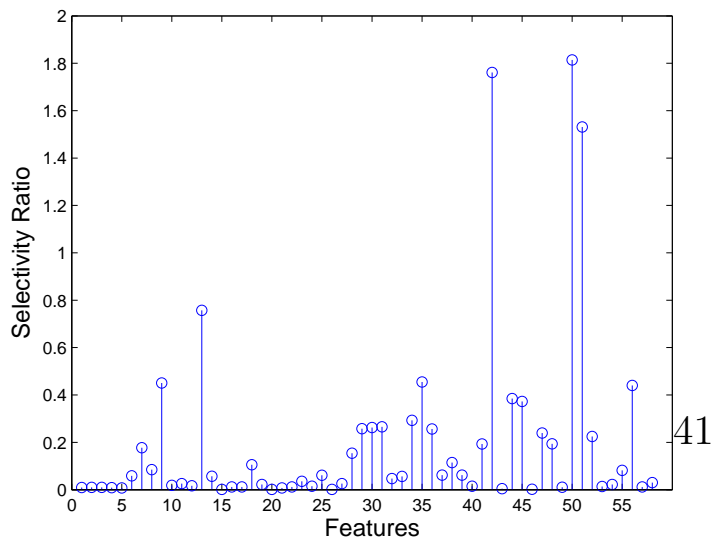

\begin{tabular}{|l|l|l|}
\hline \multicolumn{2}{|l|}{ Feature } & SR \\
\hline 50 & familiarity & 1.81 \\
42 & noun frequency & 1.76 \\
51 & noun familiarity & 1.53 \\
13 & dependent clauses/clause & 0.76 \\
\hline
\end{tabular}

